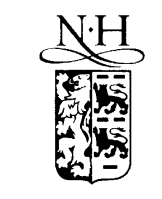

\title{
Total cross sections for positron scattering on argon and krypton at intermediate and high energies
}

\author{
Grzegorz P. Karwasz *, Mario Barozzi, Roberto S. Brusa, Antonio Zecca \\ Dipartimento di Fisica, Istituto Nazionale per la Fisica della Materia, Università di Trento, 38050 Povo (TN), Italy
}

\begin{abstract}
Preliminary total cross section data for positron scattering on argon and krypton between 250 and $5000 \mathrm{eV}$ are presented. A Ramsauer-type apparatus, previously used for electron-scattering measurements has been modified. To check for the convergence between electron and positron total cross sections, a semiempirical analysis has been applied. The analysis for argon indicates an asymptotic convergence for electron and positron projectiles - the high energy parameters of the both fits are equal within the uncertainty limits. No such a clear conclusion is possible with the present data up to $5000 \mathrm{eV}$ for krypton. (C) 2002 Elsevier Science B.V. All rights reserved.
\end{abstract}

PACS: 41.75.Fr; 34.80.Bm

\section{Introduction}

High energy measurements of positron-scattering cross sections are scarce (see [1]). The main contribution to this field was brought by the Detroit group [2,3] but their experiments stopped at $800 \mathrm{eV}$ collision energy. The only measurements extending up to $3000 \mathrm{eV}$ are those of Dutton et al. for $\mathrm{N}_{2}$ [4]. Measurements at high energies are essential for testing the convergence between electron and positron cross section as well as the dispersion relation [2]. At low energies positron elastic cross sections are, in general, lower than the electron ones due to different signs of the static and polarization interactions. For fast collisions the polarization interaction becomes less important

\footnotetext{
${ }^{*}$ Corresponding author. Address: INFM, Dipartimento di Fisica, Università di Trento, 38050 Povo $(\mathrm{TN})$, Italy. Tel.: +390461-88-1554; fax: +39-0461-88-1696.

E-mail address: karwasz@science.unitn.it (G.P. Karwasz).
}

and total cross sections for the two projectiles should coincide [1]. Measurements on noble targets showed merging at $200 \mathrm{eV}$ in $\mathrm{He}$ [2], but no merging for heavier targets $(\mathrm{Ne}, \mathrm{Ar}, \mathrm{Kr}, \mathrm{Xe})$ up to $800 \mathrm{eV}[2,3]$. On the other hand, such a merging was observed by Sueoka and collaborators at energies as low as $50 \mathrm{eV}$ in numerous molecular targets, like $\mathrm{C}_{2} \mathrm{H}_{2}, \mathrm{C}_{2} \mathrm{H}_{4}, \mathrm{SiH}_{4}, \mathrm{CCl}_{4}$ [5].

In particular, measurements from the Detroit group showed, in the high energy limit of their apparatus, a difference between electron and positron cross sections of $15 \%$ for argon [2] and $20 \%$ for krypton [3]. It results also from those works that the positron scattering on argon agrees with the dispersion relation, no agreement was found for electron-argon scattering and no conclusions were given for krypton. The dispersion relation contains total cross sections integrated up to infinite energy [2] and therefore the validity of the verification depends much on the availability of positron and electron data at high energies. In our 
previous measurements we reported electron-scattering cross sections up to $3 \mathrm{keV}$ in Ar [6] and up to $4 \mathrm{keV}$ in $\mathrm{Kr}$ [7]. Present measurements extend the upper limit of positron (and electron) data to $5000 \mathrm{eV}$ and are performed in essentially the same set-up as our earlier experiments for numerous atomic and molecular gases (see [8]).

\section{Experimental}

A Ramsauer-type apparatus [6] has been modified for positron-scattering experiment. Briefly, a uniform, perpendicular magnetic field is used to determine the geometry of the beam. The scattering cell extends to the $45^{\circ}$ sector of the field but only half of it is filled with gas. This solution improves the angular resolution of measurements. The entrance and exit apertures of the gas cell are respectively $3.2 \times 9.2$ and $7.2 \times 8.0 \mathrm{~mm}$ (width $\times$ height). The overall angular resolution of the apparatus is $1.0 \mathrm{msr}$, compared to $0.34 \mathrm{msr}$ in our electron-scattering measurements on krypton [7] and $0.16 \mathrm{msr}$ on argon [6]. The apparatus does not perform screening against inelastically scattered projectiles, apart that inherent for the use of the perpendicular magnetic field. A scheme of the scattering geometry is shown in Fig. 1.

Positrons are obtained from a $1.5 \mathrm{mCi}{ }^{22} \mathrm{Na}$ positron source and $1 \mu \mathrm{m}$ thick tungsten monocrystal moderator in a transmission geometry. Non-scattered positrons were detected by a chan-

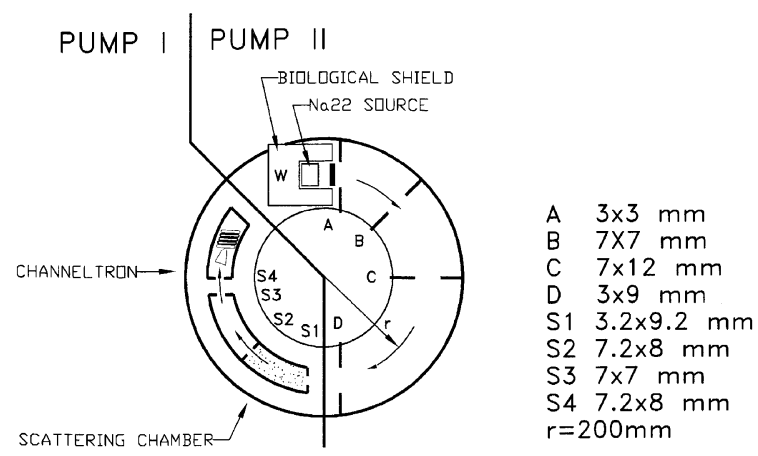

Fig. 1. A schematic drawing of the scattering cell and the beam-forming region in the present experiment. Dimensions of the slits in $\mathrm{mm}$ (width $\times$ height) are given. neltron. The average counting rate is $50 \mathrm{e}^{+} / \mathrm{s}$, lowering to $15 \mathrm{e}^{+} / \mathrm{s}$ in the low energy limit (200$300 \mathrm{eV})$. Present electron-scattering data have been obtained using secondary electrons from the moderator and not changing the remaining settings of the apparatus (apart from inverting the direction of the magnetic field). The counting rate was about $500 \mathrm{e}^{-/ \mathrm{s}}$.

The total cross section vs. collision energy $E$ has been evaluated from de Beer-Lambert's formula

$I=I_{0} \exp [-\sigma(E) L N]$,

where $I$ and $I_{0}$, respectively, are the projectile signal with and without gas in the cell, $L$ is the path length of projectiles in the gas cell $(140.2 \mathrm{~mm})$ and $N$ is the gas density. Pressures used are in the $10^{-1}$ $\mathrm{Pa}$ range, to avoid multiple scattering.

The total cross section for each energy has been determined as an average from 5-20 runs and 6-8 measurements of $I / I_{0}$ in each run. Typical statistical errors were $3-4 \%$. A special attention has been devoted to reducing the statistical error in significant energy points, like 500, 1000, $2500 \mathrm{eV}$. The systematic error of the apparatus was evaluated as $2.5 \%$ for our previous electron measurements in $\mathrm{Ar}$ [6]. In present measurements this value is probably slightly higher - no tracing of the electron current to the scattering cell is possible now. On the other hand, the overall stability of the beam intensity is better in the case of the radioactive source of electrons and/or positrons than in the case of thermoionic cathode [6]. The angular resolution error will be discussed below.

\section{Results}

Data for Ar are shown in Fig. 2. Error bars, in some cases within the point size, correspond to statistical errors solely. Present positron data for Ar below $700 \mathrm{eV}$ coincide within the overall error bar with those by Kauppila et al. [2,9]. The present electron-scattering cross section at $300 \mathrm{eV}$ coincides with our previous determination as well as with other measurements [10,11]. Above $600 \mathrm{eV}$ present electron values start to diverge with our previous determinations and with the data of 


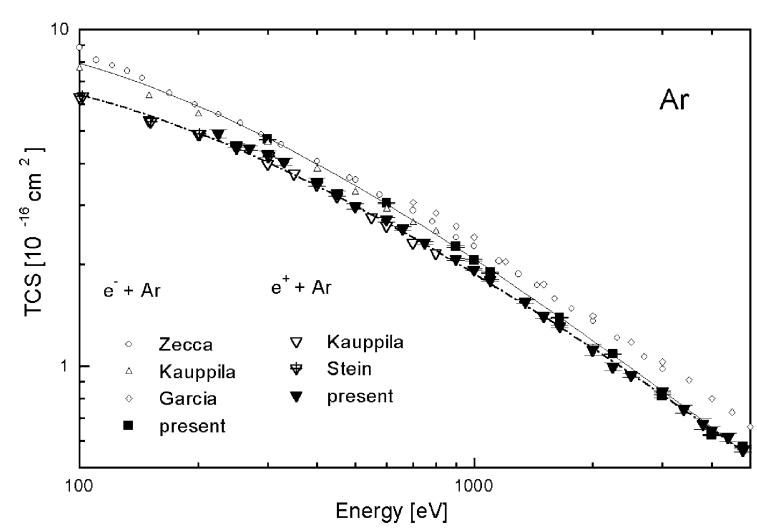

Fig. 2. Total cross sections for electron and positron scattering on argon. Trento data: Zecca et al. [6] and present. Detroit data: Kauppila et al. [2], Stein et al. [9]. Garcia et al. [12]. Lines, semiempirical fit with formula (3).

Garcia et al. [12] obtained with a much better angular resolution (of the $10^{-5} \mathrm{sr}$ order). At $3000 \mathrm{eV}$ the present value is by $26 \%$ and $20 \%$ lower than the cross sections measured by Garcia et al. [12] and Zecca et al. [6], respectively. This discrepancy is clearly due to the angular resolution error.

In order to evaluate the angular resolution error, detailed data on near-to-zero angle differential cross sections for separate processes (elastic, electronic excitation, ionization) are needed. To our knowledge, only elastic data are available from experiment [13]. Therefore, we are not able to evaluate a priori the overall angular resolution error of our measurements. To the first approximation, the Born formula predicts the differential cross section constant in angle (at near-to-zero angles) and energy (see $[8,13])$. For these reasons, we can roughly assume that the angular resolution error rises linearly with energy and with the angular acceptance of the detector. This reasoning compares well with the argon discrepancies at 3000 $\mathrm{eV}$, with a negligible error for the experiment of Garcìa et al. (say $0.25 \%$ ), a few percent for the data of Zecca et al. [6] and about 25\% presently.

Present data for krypton (see Fig. 3) are somewhat higher than the measurements from the Detroit group, with difference of $9 \%$ at $700 \mathrm{eV}$. Our lowest energy points in $\mathrm{Kr}$ can be affected by some systematical overestimation due to a poorer beam stability, however at $700 \mathrm{eV}$ the discrepancy is

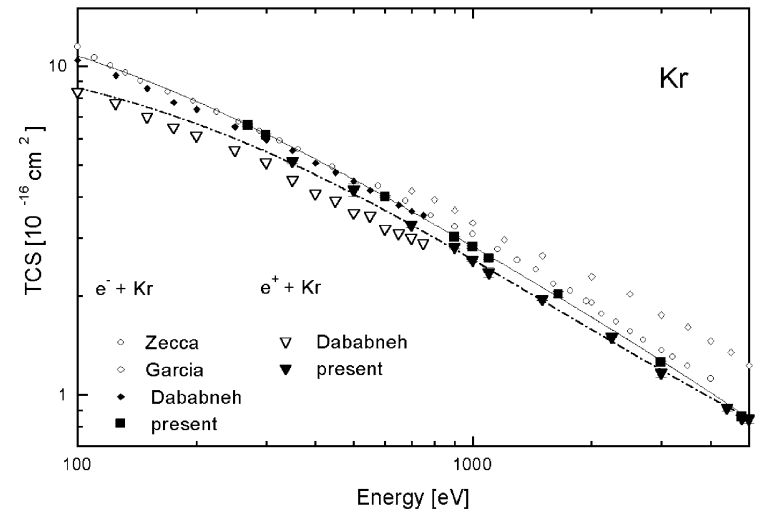

Fig. 3. Total cross sections for electron and positron scattering on krypton. Trento data: Zecca et al. [7] and present. Detroit data: Dababneh et al. [3]. Garcìa et al. [12]. Lines, semiempirical fit with formula (3).

probably to be attributed to a poorer angular resolution of the Detroit apparatus [3].

For present electron data on $\mathrm{Kr}$ similar discrepancies are present as for Ar, with the difference between previous [7] and present data rising from $0 \%$ at $300 \mathrm{eV}$ to $10 \%$ at $1000 \mathrm{eV}$. We quote $1000 \mathrm{eV}$ for comparison, as at this energy the previous electron data were still in a good agreement with other determinations [10] apart from that of Garcia et al. [12]. We note that the measurements of the latter authors, although performed with a very good angular resolution, could have suffered from some systematic error, due to an indirect way (from the optical emission intensity) of the pressure determination. If normalizing the krypton value of Garcìa et al. at $900 \mathrm{eV}$ to that of Zecca et al. [7], a conclusion about a similar underestimation of the present $\mathrm{Kr}$ and Ar data can be drawn (about 25\% at $3000 \mathrm{eV}$ ). Even though the partitioning into elastic and inelastic channels can differ somewhat for positron and electrons [1] and therefore the angular resolution errors will not be the same, our data allow for some conclusions on the electron and positron cross sections convergence.

\subsection{Convergence of positron and electron cross sections}

To compare electron and positron cross sections in the limit of high energy, we perform some 
semiempirical analysis. In the past, we have shown [14] that the following equation, based on Born formula for elastic cross section, allows to approximate total cross sections for both atomic and molecular targets in the intermediate (from a few tens of $\mathrm{eV}$ to a few $\mathrm{keV}$ ) energy range:

$$
\begin{aligned}
\sigma(E)= & \frac{1}{a(b+E)}+\frac{1}{c(d+E)} \\
& +\frac{2}{E} \sqrt{\frac{b d}{a c}} \frac{1}{|b-d|}\left[\ln \frac{\frac{E}{d}+1}{\frac{E}{b}+1}\right],
\end{aligned}
$$

with $E$ being energy and $a, b, c, d$ adjustable parameters. The advantage of using this formula, instead of the Born-Bethe one [12], is that it allows to express explicitly the cross section in two asymptotic energy limits $\sigma(E=\infty)=A / E$ and $\sigma(E=$ $0)=\sigma_{0}$, where

$$
A=\frac{1}{a}+\frac{1}{c}+\frac{2}{|b-d|} \sqrt{\frac{b d}{a c}}\left|\ln \frac{b}{d}\right|
$$

and

$\sigma_{0}=\frac{1}{a b}+\frac{1}{c d}$.

Approximating cross sections with an analytic formula allows to reduce some unavoidable spread of the data and the uncertainty on converging caused by a limited number of experimental points.

Fits to present electron and positron data are shown by lines in Figs. 2 and 3. For argon the quality of the fit is good, approximating not only present results but also previous data from the Detroit [2,9] and Trento [6] laboratories (the latter at low energies, for reasons explained before). The parameter $A$, i.e. indicating the high energy convergence, coincide for positron and electron scattering within $7 \%$, what is a reasonable limit of the overall (experimental plus semiempirical analysis) uncertainty. Parameter $\sigma_{0}$, on the other hand, are different $\left(12.3 \times 10^{-20} \mathrm{~m}^{2}\right.$ for electrons and $10.5 \times$ $10^{-20} \mathrm{~m}^{2}$ for positrons) as it is expected for different projectiles [1].

Note, that the proposed analysis does not answer the question "at which energy do the cross sections converge". In our experiment positron and electron data in argon coincide within the error bars just at $3000 \mathrm{eV}$, but up to $5000 \mathrm{eV}$ the majority of positron-cross section points still falls below the electron data. On the other hand, the semiempirical formulae apply in the whole 100$5000 \mathrm{eV}$ energy range.

We estimate the overall error of our semiempirical procedure to be about 10\% (apart from the angular resolution error). This figure takes into account the spread of the experimental data and the uncertainty levels on the choice of the fitting parameters (different combinations of the fit parameters change insignificantly the fit chi-square value). Coinciding of the $A$ parameter for positrons and electrons within $10 \%$ indicates, in our opinion, for the convergence of the cross sections. Therefore, within the range of present measurements we can state that the positron and electron cross sections in argon effectively do merge, but we cannot quote an exact figure - at which energy.

For krypton, the positron and electron formulae also converge asymptotically in the limit of $5000 \mathrm{eV}$ (see lines in Fig. 3). However, the uncertainty on the fit parameters, due to a limited number of points, is much bigger than in the case of argon - the minimum of the chi-square parameter in the approximation procedure is too shallow to allow a decisive statement on the $A$ parameters. Some more measurements for both projectiles are needed in the whole $200-5000 \mathrm{eV}$ range to judge on the convergence of the cross sections.

We recall once more, that the present analysis is only of a qualitative validity, as the present data are underestimated in their high energy limit, due to a worsened angular resolution of the apparatus if compared to our previous measurements [6]. Therefore, the high energy parameters, both for $\mathrm{e}^{+}$ and $\mathrm{e}^{-}$should be corrected upwards. The exact entity of this correction has to be evaluated theoretically or semiempirically - by performing measurements with different angular resolutions. Finally, as the partitioning schemes into elastic and inelastic channels could differ by some percent for positrons and electrons, the angular corrections would be slightly different. For this reason, an improvement of the angular resolution is planned for our future measurements. 


\section{References}

[1] W.E. Kauppila, T.S. Stein, Adv. At. Mol. Phys. 26 (1990) 1.

[2] W.E. Kauppila, T.S. Stein, J.H. Smart, M.S. Dababneh, Y.-K. Ho, V. Pol, Phys. Rev. A 24 (1981) 725.

[3] M.S. Dababneh, Y.-F. Hsieh, W.E. Kauppila, V. Pol, T.S. Stein, Phys. Rev. A 26 (1982) 1252.

[4] J. Dutton, C.J. Evans, H.L. Mansour, J. Phys. B 20 (1987) 2607.

[5] M. Kimura, O. Sueoka, A. Hamada, Y. Itikawa, Adv. Chem. Phys. 111 (2000) 537.

[6] A. Zecca, S. Oss, G. Karwasz, R. Grisenti, R.S. Brusa, J. Phys. B 20 (1987) 5157.

[7] A. Zecca, G. Karwasz, R. Grisenti, R.S. Brusa, J. Phys. B 24 (1991) 2737.
[8] G.P. Karwasz, A. Zecca, R.S. Brusa, La Rivista del Nuovo Cimento 24 (1) (2001) 1.

[9] T.S. Stein, W.E. Kauppila, C.K. Kwan, S.P. Parikh, S. Zhou, Hyperfine Interact. 73 (1992) 53.

[10] R.W. Wagenaar, F.J. de Heer, J. Phys. B 18 (1985) 2021.

[11] J.C. Nickel, K. Imre, D.F. Register, S. Trajmar, J. Phys. B 18 (1985) 125.

[12] G. Garcìa, F. Arqueros, J. Campos, J. Phys. B 19 (1986) 3777.

[13] R.H.J. Jansen, F.J. de Heer, H.J. Luyken, B. van Wingerden, H.J. Blaauw, J. Phys. B 9 (1976) 185.

[14] R.S. Brusa, G.P. Karwasz, A. Zecca, Z. Phys. D 38 (1996) 279;

A. Zecca, G.P. Karwasz, R.S. Brusa, J. Phys. B. 33 (2000) 843. 